\title{
ON $p$-HYPERELLIPTICITY OF DOUBLY SYMMETRIC RIEMANN SURFACES
}

\author{
Ewa KozeOwsKa-Walania
}

\begin{abstract}
Studying commuting symmetries of $p$-hyperelliptic Riemann surfaces, Bujalance and Costa found in [3] upper bounds for the degree of hyperellipticity of the product of commuting $(M-q)$ - and $\left(M-q^{\prime}\right)$-symmetries, depending on their separabilities. Here, we find necessary and sufficient conditions for an integer $p$ to be the degree of hyperellipticity of the product of two such symmetries, taking into account their separabilities. We also give some results concerning the existence and uniqueness of symmetries from which we obtain a series of important results of Natanzon concerning $M$ and $(M-1)$-symmetries.
\end{abstract}

\section{Introduction}

Let $X$ be a compact Riemann surface of genus $g>1$ and let $a$ be its symmetry, that is an anticonformal involution of $X$. By the classical result of Harnack, the set Fix $(a)$ of the points fixed by $a$, consists of at most $g+1$ disjoint simple curves, which are called ovals of $a$. Symmetry having $g+1$ ovals is called, following Natanzon's terminology from [6], an $M$-symmetry. If $a$ has $g+1-q$ ovals, then we shall call it an $(M-q)$-symmetry. In his work S. M. Natanzon studied symmetries of 0-hyperelliptic and 1-hyperelliptic Riemann surfaces; such surfaces are called hyperelliptic and elliptic-hyperelliptic, respectively. Some of his results can be found in $[\mathbf{2}]$ as well as in [6].

A symmetry $a$ of $X$ is called separating if $X \backslash \operatorname{Fix}(a)$ has two components and non-separating if $X \backslash \operatorname{Fix}(a)$ has one component. By saying that a symmetry has given separability, we shall understand that it is

2000 Mathematics Subject Classification. Primary: 30F; Secondary: $14 \mathrm{H}$.

Key words. Riemann surface, symmetry of Riemann surface, oval of a symmetry of a Riemann surface.

Supported by BW 5100-5-0198-6. 
specified, if the symmetry is separating or not. The surface $X$ is $p$-hyperelliptic if $X$ admits a conformal involution $\rho$ such that its orbit space $X / \rho$ has genus $p$. Such involution is called a $p$-hyperelliptic involution.

In [3] upper bounds for the degree $p$ of hyperellipticity of the product of commuting $(M-q)$ - and $\left(M-q^{\prime}\right)$-symmetries, depending on their separabilities, in terms of $q$ and $q^{\prime}$ were found. On the other hand, from Theorem 3 in $[\mathbf{1}]$, it follows that for $g \geq q+q^{\prime}+1$ such symmetries commute. In this work we shall study the $p$-hyperellipticity of the product of two $(M-q)$ - and $\left(M-q^{\prime}\right)$-symmetries of a Riemann surface $X$ of genus $g$ for $g, q, q^{\prime}$ satisfying the relation $g \geq q+q^{\prime}+1$. In such situation we find in Proposition 3.6 a better upper bound for $p$ in case of two non-separating symmetries. In Propositions 3.4-3.6 we find lower bounds for $p$ in terms of $q$ and $q^{\prime}$ depending on separability of each of the symmetries and in Theorems 3.7-3.9 we show that for arbitrary $p$ in ranges given before, there exists a Riemann surface of genus $g$ having two $(M-q)$ - and $\left(M-q^{\prime}\right)$-symmetries with specified separabilities, whose product is a $p$-hyperelliptic involution.

Finally in the last section we give some results concerning the existence and uniqueness of symmetries, from which we obtain new proofs of important results of Natanzon [6] concerning $M$ - and ( $M-1)$-symmetries. In particular, in Theorem 4.1 we prove that a Riemann surface of genus $g>4 q+1$ has at most two $(M-q)$-symmetries and that their product is a $p$-hyperelliptic involution for some $p$ in range $0 \leq p \leq 2[q / 2]$, where $[\cdot]$ denotes the function of integer part and this theorem gives Natanzon's results as corollaries. Crucial to the proof of Theorem 4.1 are the results of Tyszkowska from [8], where there are given necessary and sufficient conditions on $p, q$ and $g$ for the existence of a $p q$-hyperelliptic Riemann surface $X$ (that is the Riemann surface which is both $p$ - and $q$-hyperelliptic) of genus $g$ and also some conditions concerning uniqueness and commutativity of $p$ - and $q$-hyperelliptic involutions of $X$.

The author is grateful to the referee for helpful comments and suggestions concerning the first version of this paper.

\section{Preliminaries}

We shall prove our results using theory of non-euclidean crystallographic groups ( $N E C$-groups in short) by which we mean discrete and co-compact subgroups of the group $\mathcal{G}$ of all isometries of the hyperbolic plane $\mathcal{H}$. The algebraic structure of such group $\Lambda$ is determined by the 
signature:

(1) $s(\Lambda)=\left(g ; \pm ;\left[m_{1}, \ldots, m_{r}\right] ;\left\{\left(n_{11}, \ldots, n_{1 s_{1}}\right), \ldots,\left(n_{k 1}, \ldots, n_{k s_{k}}\right)\right\}\right)$, where the brackets $\left(n_{i 1}, \ldots, n_{i s_{i}}\right)$ are called the period cycles, the integers $n_{i j}$ are the link periods, $m_{i}$-proper periods and finally $g$ the orbit genus of $\Lambda$.

A group $\Lambda$ with signature (1) has the presentation with the following generators, called canonical generators:

(i) $x_{i}, i=1, \ldots, r$,

(ii) $c_{i j}, i=1, \ldots, k, j=0, \ldots, s_{i}$,

(iii) $e_{i}, i=1, \ldots, k$,

(iv) $a_{i}, b_{i}, i=1, \ldots, g$ if the sign is + , $d_{i}, i=1, \ldots, g$, if the sign is -

and relations:

(i) $x_{i}^{m_{i}}=1, i=1, \ldots, r$,

(ii) $c_{i j}^{2}=\left(c_{i j-1} c_{i j}\right)^{n_{i j}}=1, i=1, \ldots, k, j=1, \ldots, s_{i}$,

(iii) $c_{i 0}=e_{i}^{-1} c_{i s_{i}} e_{i}, i=1, \ldots, k$,

(iv) $x_{1} \ldots x_{r} e_{1} \ldots e_{k}\left[a_{1}, b_{1}\right] \ldots\left[a_{g}, b_{g}\right]=1$, if the sign is + , $x_{1} \ldots x_{r} e_{1} \ldots e_{k} d_{1}^{2} \ldots d_{g}^{2}=1$ if the sign is -.

The elements $x_{i}$ are elliptic transformations, $a_{i}, b_{i}$-hyperbolic translations, $d_{i}$-glide reflections, $c_{i j}$-hyperbolic reflections and reflections $c_{i j-1}$ and $c_{i j}$ are said to be consecutive.

Now an abstract group with such presentation can be realized as an NEC-group $\Lambda$ if and only if the value

$$
2 \pi\left(\varepsilon g+k-2+\sum_{i=1}^{r}\left(1-\frac{1}{m_{i}}\right)+\frac{1}{2} \sum_{i=1}^{k} \sum_{j=1}^{s_{i}}\left(1-\frac{1}{n_{i j}}\right)\right),
$$

where $\varepsilon=2$ or 1 according to the sign being + or - , is positive. This value turns out to be the hyperbolic area $\mu(\Lambda)$ of an arbitrary fundamental region for such group and we have the following Hurwitz-Riemann formula

$$
\left[\Lambda: \Lambda^{\prime}\right]=\mu\left(\Lambda^{\prime}\right) / \mu(\Lambda)
$$

for a subgroup $\Lambda^{\prime}$ of finite index in an NEC-group $\Lambda$.

NEC-groups having no orientation reversing elements are classical co-compact Fuchsian groups. They have signatures being of the form $\left(g ;+;\left[m_{1}, \ldots, m_{r}\right] ;\{-\}\right)$, which shall be abbreviated as $\left(g ; m_{1}, \ldots, m_{r}\right)$. Given an NEC-group $\Lambda$, the subgroup $\Lambda^{+}$of $\Lambda$ consisting of all the 
orientation-preserving elements is called the canonical Fuchsian subgroup of $\Lambda$ and for a group with signature (1) it has, by [7], signature

$$
\left(\varepsilon g+k-1 ; m_{1}, m_{1}, \ldots, m_{r}, m_{r}, n_{11}, \ldots, n_{k s_{k}}\right) .
$$

A torsion free co-compact Fuchsian group $\Gamma$ is called a surface group and it has signature $(g ;-)$. In such case $\mathcal{H} / \Gamma$ is a compact Riemann surface of genus $g$ and conversely, each compact Riemann surface can be represented as such orbit space for some $\Gamma$. Furthermore, given a Riemann surface so represented, a finite group $G$ is a group of automorphisms of $X$ if and only if $G=\Lambda / \Gamma$ for some NEC-group $\Lambda$ containing $\Gamma$ as a normal subgroup.

The following two results follow easily from [4] and are crucial for the paper.

Lemma 2.1 (c.f. Theorems 2.3.2, 2.3.3). Let $\Lambda$ be an NEC-group with the signature (1), let $\Lambda^{\prime}$ be a normal subgroup of index $N$ and assume that no pair of consecutive canonical reflections of $\Lambda$ belongs to $\Lambda^{\prime}$. Then $\Lambda^{\prime}$ has only empty period cycles. Furthermore each canonical reflection $c_{i j}$ of $\Lambda$ corresponding to a period cycle $C_{i}=\left(n_{i 1}, \ldots, n_{i s_{i}}\right)$ which is in $\Lambda^{\prime}$ produces in the last either $N / n(i)$ empty period cycles, where $n(i)$ is the order of the image in $\Lambda / \Lambda^{\prime}$ of $e_{i}$ if $C_{i}$ is empty and $N / 2 n(i, j)$ empty period cycles, where $n(i, j)$ is the order of the image in $\Lambda / \Lambda^{\prime}$ of $c_{i j-1} c_{i j+1}$ and all period cycles of $\Lambda^{\prime}$ arise in this way.

Let $\Lambda^{\prime}$ be a normal subgroup of an NEC-group $\Lambda$. A canonical generator of $\Lambda$ is proper (with respect to $\Lambda^{\prime}$ ) if it does not belong to $\Lambda^{\prime}$. The elements of $\Lambda$ expressable as a composition of proper generators of $\Lambda^{\prime}$ are the words of $\Lambda$ (with respect to $\Lambda^{\prime}$ ). With these notations we have

Lemma 2.2 (c.f. Theorem 2.1.3). Let us suppose that $\left[\Lambda: \Lambda^{\prime}\right]$ is even and $\Lambda$ has sign + . Then $\Lambda^{\prime}$ has sign + if and only if no orientation reversing word belongs to $\Lambda^{\prime}$. If $\left[\Lambda: \Lambda^{\prime}\right]$ is even and $\Lambda$ has the sign - , then $\Lambda^{\prime}$ has the sign - if and only if either a glide reflection of the canonical generators of $\Lambda$ or an orientation reversing word belongs to $\Lambda^{\prime}$.

\section{Pairs of symmetries of Riemann surfaces}

Let $X$ be a Riemann surface of genus $g \geq 2$, having an $(M-q)$-symmetry $a$ and an $\left(M-q^{\prime}\right)$-symmetry $b$, different from $a$, for $g \geq q+q^{\prime}+1$. From Theorem 3 in $[\mathbf{1}]$ we know that in this case these symmetries commute. Now $X=\mathcal{H} / \Gamma, a$ and $b$ are the images of orientation reversing elements of $\Lambda$ and $G=Z_{2} \oplus Z_{2}=\langle a, b\rangle=\Lambda / \Gamma$ for some Fuchsian surface 
group $\Gamma$ being a normal subgroup of an NEC-group $\Lambda$ with signature

$$
\left(h ; \pm ;[2, . r ., 2] ;\left\{\left(2, . s_{1} ., 2\right), \ldots,\left(2, s_{k} ., 2\right),(-), . . . .,(-)\right\}\right) .
$$

Let $\theta: \Lambda \rightarrow G=\Lambda / \Gamma$ denote the canonical projection and let $\Gamma_{a}$ and $\Gamma_{b}$ be the inverse images of the groups generated by $a$ and $b$, respectively. These are the subgroups of $\Lambda$ of index 2. By (2) they have neither proper periods nor link periods and the separabilities of $a$ and $b$ depend on the sign in signatures of $\Gamma_{a}$ and $\Gamma_{b}$ respectively; for the sign + the corresponding symmetry is separating and for the sign - it is a nonseparating one. Furthermore, the number of ovals of $a$ and $b$ equals the number of empty period cycles in $\Gamma_{a}$ and $\Gamma_{b}$, respectively. Observe finally, that the product $a b$ is a $p$-hyperelliptic involution, where $p$ is the orbit genus of $\Lambda^{+}$.

Lemma 3.1. A nonempty period cycle of $\Lambda$ has even length $s$ and it contributes $s / 2$ ovals to each of symmetries.

Proof: Indeed, for a period cycle $C=(2, . \stackrel{s}{.}, 2)$ we have corresponding canonical generators $c_{0}, \ldots, c_{s}, e$. Then there are $\lambda_{1}, \lambda_{2} \in \Lambda \backslash \Lambda^{+}$ for which $\theta\left(\lambda_{1}\right)=a$ and $\theta\left(\lambda_{2}\right)=b$. So none of $c_{i}$ is mapped onto $a b$ since otherwise $\lambda_{1} \lambda_{2} c_{i}$ would be an orientation reversing element of $\Gamma$. Similarly $\theta\left(c_{i}\right) \neq \theta\left(c_{i+1}\right)$ since otherwise $c_{i} c_{i+1}$ would be an element of order 2 in $\Gamma$. So our epimorphism sends the generators $c_{i}$ alternatively to $a$ and $b$. Furthermore $s$ must be even (otherwise $a b=1$, a contradiction) and by Lemma 2.1, $\Gamma_{a}$ and $\Gamma_{b}$ have $s / 2$ empty period cycles proceeding from $C$.

Lemma 3.2. Let $X=\mathcal{H} / \Gamma$ be a Riemann surface of genus $g$ having two commuting symmetries, let $G=\Lambda / \Gamma$ be the group generated by these symmetries and let $\theta: \Lambda \rightarrow G$ denote the canonical projection. Then, the following conditions hold:

(1) Both symmetries are separating if and only if $\operatorname{sgn}(\Lambda)=+, r=0$ and $\theta\left(e_{i}\right)=1$ for all $i$;

(2) Just one of the symmetries is separating if and only if $\operatorname{sgn}(\Lambda)=-$, $r=0, \theta\left(e_{i}\right)=1$ for all $i$ and $\theta\left(d_{1}\right)=\cdots=\theta\left(d_{h}\right)$

(3) Both symmetries are non-separating if and only if one of the following holds
(a) $\operatorname{sgn}(\Lambda)=-, h>1, \theta\left(d_{i}\right) \neq \theta\left(d_{j}\right)$ for some $i \neq j$;
(b) $r>0$;
(c) $\theta\left(e_{i}\right) \neq 1$ for some $i$. 
Proof: Let $a$ and $b$ be two commuting symmetries of a Riemann surface $X$ and let $\lambda$ and $\lambda^{\prime}$ be two orientation reversing elements of $\Lambda$ such that $\theta(\lambda)=a$ and $\theta\left(\lambda^{\prime}\right)=b$.

Assume first that both $a$ and $b$ are separating. It is easy to see that $\operatorname{sgn}(\Lambda)=+$. Indeed, if otherwise, we would have a canonical glide reflection, say $d$, and without loss of generality we can assume that $\theta(d)=a$. Now we have a glide reflection in $\Gamma_{a}$ and so it has the sign -. Thus $a$ is non-separating, a contradiction. If $r>0$ we would have an elliptic generator $x$ and $\theta(x)=a b$. Now obviously $\lambda^{\prime}$ and $x$ are not in $\Gamma_{a}$ and so $\lambda^{\prime} x$ is an orientation reversing word in $\Gamma_{a}$ and therefore $a$ is non-separating by Lemma 2.2 , a contradiction. Similarly, if $\theta\left(e_{i}\right) \neq 1$ for some $i$, we would have an orientation reversing word in $\Gamma_{a}$.

Conversely, if $\operatorname{sgn}(\Lambda)=+, r=0, \theta\left(e_{i}\right)=1$ for all $i$, then both $a$ and $b$ must be separating because no orientation reversing word belongs either to $\Gamma_{a}$ or to $\Gamma_{b}$.

Assume now that $a$ is separating and $b$ is non-separating. It is easy to see that it must be $r=0, \theta\left(e_{i}\right)=1$ for all $i$. Indeed, otherwise as before we would have an orientation reversing word $\lambda^{\prime} x$ or $\lambda^{\prime} e_{i}$ in $\Gamma_{a}$ and so $a$ would be non-separating. Now if $\operatorname{sgn}(\Lambda)=+$, we already know that both symmetries would be separating, so it must be $\operatorname{sgn}(\Lambda)=-$. Thus we only have to show that $\theta\left(d_{1}\right)=\cdots=\theta\left(d_{h}\right)$ for the canonical glide reflections of $\Lambda$. Indeed if $\theta\left(d_{i}\right) \neq \theta\left(d_{j}\right)$ for some $i \neq j$ then $\theta\left(d_{i}\right)=a$, $\theta\left(d_{j}\right)=b$ and so both $a$ and $b$ are non-separating by Lemma 2.2.

Conversely, let $\operatorname{sgn}(\Lambda)=-, r=0, \theta\left(e_{i}\right)=1$ for all $i$ and $\theta\left(d_{1}\right)=$ $\cdots=\theta\left(d_{h}\right)$. Without loss of generality we can assume $\theta\left(d_{i}\right)=b$ for all $i$. By Lemma 2.2, $b$ is non-separating as we have a glide reflection in $\Gamma_{b}$. Symmetry $a$ is separating because no orientation reversing word belongs to $\Gamma_{a}$.

Let finally $a$ and $b$ be both non-separating. If none of conditions required in theorem holds, we have two possible cases: $\{\operatorname{sgn}(\Lambda)=+$, $r=0, \theta\left(e_{i}\right)=1$ for all $\left.i\right\}$ or $\left\{\operatorname{sgn}(\Lambda)=-, r=0, \theta\left(e_{i}\right)=1\right.$ for all $i$ and $h=1\}$. In the first case obviously both symmetries would be separating by the first part of the proof. In the second case only one of the symmetries would be separating, because condition $\theta\left(d_{1}\right)=\cdots=$ $\theta\left(d_{h}\right)$ holds with $h=1$.

The converse follows from the previous part of the proof.

Lemma 3.3. If $(M-q)$-symmetry of a Riemann surface $X$ of genus $g$ is separating, $q$ must be even. If $X$ has also $\left(M-q^{\prime}\right)$-symmetry and the symmetries commute, then also $q^{\prime}$ must be even. 
Proof: First part of the proof is a direct consequence of a theorem due to Harnack which says that,

$$
g+1 \equiv g+1-q \bmod 2
$$

and thus $q \equiv 0 \bmod 2$.

We know that $G=\Lambda / \Gamma$ for some NEC-group $\Lambda$ having signature (3). Now, by Lemma 3.2, $r=0$ and, if the product of both symmetries is a $p$-hyperelliptic involution, then

$$
\begin{aligned}
\pi(g-1) & =\mu(\Lambda) \\
& =2 \pi\left(\varepsilon h+l+k-2+s_{1} / 4+\cdots+s_{k} / 4\right) \\
& =2 \pi\left(p-1+s_{1} / 4+\cdots+s_{k} / 4\right)
\end{aligned}
$$

and so $s_{1}+\cdots+s_{k}=2 g+2-4 p$. Furthermore, all empty period cycles must contribute two ovals to our symmetries, since $\theta\left(e_{i}\right)=1$ for all $i$ by Lemma 3.2. Thus, the sum of ovals of both symmetries is $2 g+2-q-q^{\prime}=s_{1}+\cdots+s_{k}+4 p-q-q^{\prime}$. As a consequence of Lemma 3.1, and the fact that $q$ is even, we have that $q^{\prime}$ is also even.

In the following propositions we assume that $a$ and $b$ are $(M-q)$ - and $\left(M-q^{\prime}\right)$-symmetries of a Riemann surface $X$ of genus $g \geq q+q^{\prime}+1$. Therefore, we will be able to assume that $a$ and $b$ commute, $G=\langle a, b\rangle=$ $\Lambda / \Gamma$ for an NEC-group $\Lambda$ having signature (3) and $a b$ is a $p$-hyperelliptic involution. Assume also that $q \leq q^{\prime}$.

Proposition 3.4. If $a$ and $b$ are separating symmetries, then $q$ and $q^{\prime}$ are even and $q^{\prime} / 2 \leq p \leq q / 2+q^{\prime} / 2$ or $q^{\prime} / 2 \leq p \leq q / 2+q^{\prime} / 2+1$ for $g>q+q^{\prime}+1$ or $g=q+q^{\prime}+1$ respectively.

Proof: The upper bound for $g>q+q^{\prime}+1$ was found in [3] so we only have to show the lower bound in both cases and the upper bound for $g=q+q^{\prime}+1$.

Because both symmetries are separating, from Lemma 3.2 we know that $r=0, \theta\left(e_{i}\right)=1$ for all $i$ and $\operatorname{sgn}(\Lambda)=+$. Also we know that $p=2 h+l+k-1$. So

$$
\begin{aligned}
\pi(g-1) & =\mu(\Lambda) \\
& =2 \pi\left(2 h+l+k-2+s_{1} / 4+\cdots+s_{k} / 4\right) \\
& =2 \pi\left(p-1+s_{1} / 4+\cdots+s_{k} / 4\right) .
\end{aligned}
$$

Therefore $s_{1} / 2+\cdots+s_{k} / 2=g+1-2 p$. By Lemma 3.1 we have $g+1-2 p \leq g+1-q^{\prime}$ and so $p \geq q^{\prime} / 2$. 
The upper bound for $g=q+q^{\prime}+1$ follows from

$$
\begin{aligned}
\pi(g-1) & =\mu(\Lambda) \\
& =2 \pi\left(2 h+l+k-2+s_{1} / 4+\cdots+s_{k} / 4\right) \\
& \geq \pi\left(4 h+l+2 k-4+g+1-q / 2-q^{\prime} / 2\right) \\
& \geq \pi\left(2 h+l+k-3+g-q / 2-q^{\prime} / 2\right)
\end{aligned}
$$

as $p=2 h+l+k-1$.

Proposition 3.5. If only one of $a$ and $b$ is separating, then $q^{\prime} / 2 \leq p \leq$ $q / 2+q^{\prime} / 2-1$.

Proof: The lower bound follows directly from Lemma 3.2 and previous proposition, because also in this case $r=0$. The upper bound was found in $[3]$.

Proposition 3.6. If $a$ and $b$ are non-separating, then $\left|q^{\prime}-q\right| / 2 \leq p \leq$ $q / 2+q^{\prime} / 2-1$.

Proof: In $[\mathbf{3}]$ it was shown that for two commuting symmetries of a Riemann surface $X$ of genus $g$, such that their product is a $p$-hyperelliptic involution, we have $p<q / 2+q^{\prime} / 2+1$. Here we show first that there is a sharper bound $p<q / 2+q^{\prime} / 2$.

We first prove that $k>0$. In fact, since $a$ and $b$ are non-separating, by Lemma 3.2 we have that $h>1$ or $r>0$ or $\theta\left(e_{i}\right) \neq 1$ for some $i$. Now if $k=0$, then $\pi(g-1)=\mu(\Lambda)>2 \pi(l-2)$ in the first two cases. So $l<(g+3) / 2$ which gives that our symmetries have at most $g+3$ ovals. However, this means that $2 g+2-q-q^{\prime}<g+3$ and so $g<q+q^{\prime}+1$, which is not our case. If $h=0, r=0$ and $\theta\left(e_{i}\right) \neq 1$ for some $i$, then by Lemma 2.1 there is a period cycle, which gives less than two ovals to one of our symmetries. Thus $2 g+2-q-q^{\prime}<2 l \leq g+3$, since $\pi(g-1)=\mu(\Lambda) \geq 2 \pi(l-2)$. This gives $g<q+q^{\prime}+1$, which is not our case.

Since both symmetries are non-separating, one of three conditions in Lemma 3.2 holds. Assume first that $\operatorname{sgn}(\Lambda)=-, h>1, \theta\left(d_{i}\right) \neq \theta\left(d_{j}\right)$ for some $i \neq j$. By Lemmata 2.1 and 3.1, $2 l+s_{1}+\cdots+s_{k} \geq 2 g+2-q-q^{\prime}$ 
and so

$$
\begin{aligned}
\pi(g-1) & =\mu(\Lambda) \\
& \geq 2 \pi\left(h+l+k-2+s_{1} / 4+\cdots+s_{k} / 4\right) \\
& \geq 2 \pi\left(h+l / 2+k-2+\left(g+1-q / 2-q^{\prime} / 2\right) / 2\right) \\
& >\pi\left(h+1+l+k-2+g-q / 2-q^{\prime} / 2\right) \\
& \geq \pi\left(p+g-q / 2-q^{\prime} / 2\right)
\end{aligned}
$$

Therefore we have

$$
p<q / 2+q^{\prime} / 2-1 \text {. }
$$

If the condition $3(\mathrm{~b})$ from Lemma 3.2 holds, then

$$
\begin{aligned}
\pi(g-1) & =\mu(\Lambda) \\
& >2 \pi\left(\varepsilon h+l+k-2+s_{1} / 4+\cdots+s_{k} / 4\right) \\
& \geq 2 \pi\left(\varepsilon h+l / 2+k-2+\left(g+1-q / 2-q^{\prime} / 2\right) / 2\right) \\
& \geq \pi\left(\varepsilon h+l+k-2+g-q / 2-q^{\prime} / 2\right) \\
& \geq \pi\left(p-1+g-q / 2-q^{\prime} / 2\right)
\end{aligned}
$$

thus

$$
p<q / 2+q^{\prime} / 2 \text {. }
$$

Let finally $\theta\left(e_{i}\right) \neq 1$ for some $i, r=0$ (if $r>0$ then automatically we have our bound from previous proposition). It is obvious that $p \leq q / 2+$ $q^{\prime} / 2$ because, by Lemmata 2.1 and $3.1,2 l+s_{1}+\cdots+s_{k} \geq 2 g+2-q-q^{\prime}$ and so

$$
\begin{aligned}
\pi(g-1) & =\mu(\Lambda) \\
& =2 \pi\left(\varepsilon h+l+k-2+r / 2+s_{1} / 4+\cdots+s_{k} / 4\right) \\
& \geq \pi\left(2 \varepsilon h+l+2 k-4+r+g+1-q / 2-q^{\prime} / 2\right) \\
& \geq \pi\left(\varepsilon h+l+k-2+g-q / 2-q^{\prime} / 2\right),
\end{aligned}
$$

where $\varepsilon=2$ if the sign is + and $\varepsilon=1$ otherwise. The last inequality in (4) is true since $k>0$, as it was shown at the beginning of the proof. So $p=\varepsilon h+l+k-1 \leq q / 2+q^{\prime} / 2$ which means that the product of $(M-q)$ and $\left(M-q^{\prime}\right)$-symmetry is a $p$-hyperelliptic involution for $p \leq q / 2+q^{\prime} / 2$. Assume that $p=q / 2+q^{\prime} / 2$. Observe that we may assume $k=1$ and $h=0$ since otherwise the resulting inequality in (4) is strict. Also all empty period cycles must contribute two ovals to our symmetries. So $\theta\left(e_{i}\right)=1$ for all generators $e_{i}$ corresponding to empty period cycles. 
As $k=1$ and $r=0$ we have a relator $e_{1} \ldots e_{l+1}$ in the presentation of $\Lambda$. Now $\theta\left(e_{i}\right)=1$ for $i=1, \ldots, l$. Obviously $\theta\left(e_{1} \ldots e_{l+1}\right)=1$. So it must be $\theta\left(e_{l+1}\right)=1$ for a generator $e_{l+1}$ corresponding to the unique nonempty period cycle, a contradiction.

The lower bound follows from the fact that nonempty period cycle gives to both symmetries the same number of ovals. Indeed, as $k>0$ and $p=\varepsilon h+l+k-1 \geq l$, we have $q^{\prime}-q=(g+1-q)-\left(g+1-q^{\prime}\right) \leq 2 l \leq 2 p$ which gives $p \geq\left|q^{\prime}-q\right| / 2$.

The next theorems show that every value of $p$ within the ranges given in the previous propositions, can be attained. In order to simplify each theorem's proof we shall employ the following conventions throughout. First $q \leq q^{\prime}$ and $G=\mathrm{Z}_{2} \oplus \mathrm{Z}_{2}=\langle a, b\rangle$. An epimorphism $\theta: \Lambda \rightarrow G$ will be defined on canonical reflections $c_{0}, \ldots, c_{s}$ of $\Lambda$ corresponding to nonempty period cycle $(2, . s ., 2)$ sending them alternatively to $a$ and $b$ starting with $a$ i.e.

$$
\theta\left(c_{i}\right)= \begin{cases}a & \text { for } i=2 j, j=0, \ldots, s / 2, \\ b & \text { for } i=2 j+1, j=0, \ldots, s / 2-1\end{cases}
$$

and $\theta\left(x_{i}\right)=a b$ for arbitrary canonical elliptic element $x_{i}$ of $\Lambda$.

Theorem 3.7. Given $g \geq 2, q, q^{\prime}$ and $p$ such that $g \geq q+q^{\prime}+1$, q, $q^{\prime}$ are even and $q^{\prime} / 2 \leq p \leq q / 2+q^{\prime} / 2$, there exists a Riemann surface of genus $g$ having separating $(M-q)$ - and $\left(M-q^{\prime}\right)$-symmetries, whose product is a p-hyperelliptic involution. In addition for $g=q+q^{\prime}+1$ there exists such configuration of symmetries for $p=q / 2+q^{\prime} / 2+1$.

Proof: Let first $g \geq q+q^{\prime}+1$ and $p \leq q / 2+q^{\prime} / 2$. For $p \equiv\left(q+q^{\prime}\right) / 2$ mod 2 consider an NEC-group $\Lambda$ with signature

$$
\left(h ;+;[-] ;\left\{(-)^{l},(2, . s ., 2)\right\}\right),
$$

where $l=2 p-\left(q+q^{\prime}\right) / 2, s=2(g+1-2 p), h=\left(q+q^{\prime}-2 p\right) / 4$. Then an epimorphism $\theta: \Lambda \rightarrow G$ defined by: $\theta\left(e_{i}\right)=1$ for all $i$,

$$
\theta\left(c_{i 0}\right)= \begin{cases}a & \text { for } i=1, \ldots, p-q / 2, \\ b & \text { for } i=p-q / 2+1, \ldots, l,\end{cases}
$$

gives rise to a configuration of separating $(M-q)$ - and $\left(M-q^{\prime}\right)$-symmetries whose product is a $p$-hyperelliptic involution by Lemmata 2.1 and 3.2 .

For $p \equiv\left(q+q^{\prime}\right) / 2+1 \bmod 2$ consider an NEC-group $\Lambda$ with signature

$$
\left(h ;+;[-] ;\left\{(-)^{l},(2,2),(2, . s ., 2)\right\}\right),
$$


for $h=\left(q+q^{\prime}-2-2 p\right) / 4, s=2(g-2 p)$ and $l=2 p-\left(q+q^{\prime}\right) / 2$. Then an epimorphism $\theta: \Lambda \rightarrow G$ defined by: $\theta\left(e_{i}\right)=1$ for all $i$ and

$$
\theta\left(c_{i 0}\right)= \begin{cases}a & \text { for } i=1, \ldots, p-q / 2 \\ b & \text { for } i=p-q / 2+1, \ldots, l\end{cases}
$$

gives rise to a configuration of separating $(M-q)$ - and $\left(M-q^{\prime}\right)$-symmetries whose product is a $p$-hyperelliptic involution by Lemmata 2.1 and 3.2 .

Finally for $g=q+q^{\prime}+1, p=q / 2+q^{\prime} / 2+1$ consider an NEC-group $\Lambda$ with signature

$$
\left(0 ;+;[-] ;\left\{(-)^{p+1}\right\}\right) \text {. }
$$

Then an epimorphism $\theta: \Lambda \rightarrow G$ defined by: $\theta\left(e_{i}\right)=1$ for all $i$,

$$
\theta\left(c_{i 0}\right)= \begin{cases}a & \text { for } i=1, \ldots, q / 2+1, \\ b & \text { for } i=q / 2+2, \ldots, q / 2+q^{\prime} / 2+2=p+1,\end{cases}
$$

gives rise to a configuration of separating $(M-q)$ - and $\left(M-q^{\prime}\right)$-symmetries whose product is a $p$-hyperelliptic involution by Lemmata 2.1 and 3.2.

Theorem 3.8. Given $g \geq 2, q$, $q^{\prime}$ and $p$ such that $g \geq q+q^{\prime}+1, q$, $q^{\prime}$ even and $q^{\prime} / 2 \leq p \leq q / 2+q^{\prime} / 2-1$, there exists a Riemann surface of genus $g$ having $(M-q)$ - and $\left(M-q^{\prime}\right)$-symmetries, whose product is a p-hyperelliptic involution and just one of them is separating.

Proof: Consider an NEC-group $\Lambda$ with signature

$$
\left(h ;-;[-] ;\left\{(-)^{l},(2, . s ., 2)\right\}\right),
$$

where $l=2 p-\left(q+q^{\prime}\right) / 2, s=2(g+1-2 p), h=\left(q+q^{\prime}\right) / 2-p$. Then an epimorphism $\theta: \Lambda \rightarrow G$ defined by the assignment: $\theta\left(e_{i}\right)=1, \theta\left(d_{j}\right)=a$ for all $i, j$ and

$$
\theta\left(c_{i 0}\right)= \begin{cases}a & \text { for } i=1, \ldots, p-q / 2 \\ b & \text { for } i=p-q / 2+1, \ldots, l\end{cases}
$$

gives rise to a configuration $(M-q)$ - and $\left(M-q^{\prime}\right)$-symmetries whose product is a $p$-hyperelliptic involution and only one of symmetries is separating by Lemmata 2.1 and 3.2.

Theorem 3.9. Given $g \geq 2, q, q^{\prime}$ and $p$ such that $g \geq q+q^{\prime}+1$ and $\left|q^{\prime}-q\right| / 2 \leq p<q / 2+q^{\prime} / 2$, there exists a Riemann surface of genus $g$ having two non-separating $(M-q)$ - and $\left(M-q^{\prime}\right)$-symmetries whose product is a p-hyperelliptic involution. 
Proof: Let $q \leq q^{\prime}$ and assume first that both $\left(q^{\prime}-q\right) / 2$ and $p$ are even. So we have $q^{\prime}-q=4 \alpha$ for some integer $\alpha$. Consider an NEC-group $\Lambda$ with signature

$$
\left(0 ;+;[2, . r ., 2] ;\left\{(-)^{p},(2, . s ., 2)\right\}\right),
$$

where $r=\left(q+q^{\prime}\right) / 2-p, s=2\left(g+1-p-q / 2-q^{\prime} / 2\right)$. Then an epimorphism $\theta: \Lambda \rightarrow G$ defined by the assignment: $\theta\left(e_{i}\right)=1$ for $i=$ $1, \ldots, p, \theta\left(e_{p+1}\right)=(a b)^{r}$ and

$$
\theta\left(c_{i 0}\right)= \begin{cases}a & \text { for } i=1, \ldots, p / 2-\left(q^{\prime}-q\right) / 4, \\ b & \text { for } i=p / 2-\left(q^{\prime}-q\right) / 4+1, \ldots, p,\end{cases}
$$

gives rise to a configuration of $(M-q)$ - and $\left(M-q^{\prime}\right)$-symmetries whose product is a $p$-hyperelliptic involution. Both symmetries are non-separating as $r>0$ in virtue of $p<q / 2+q^{\prime} / 2$.

Let now $\left(q^{\prime}-q\right) / 2$ be even and $p$ odd. Assume first that $q<q^{\prime}$ or $q=q^{\prime}=p+1 \geq 4$ with $q$ even. Consider an NEC-group $\Lambda$ with signature

$$
\left(0 ;+;[2, . r ., 2] ;\left\{(-)^{p},(2, . s ., 2)\right\}\right)
$$

for $r=\left(q^{\prime}+q-2\right) / 2-p, s=2\left(g+2-p-\left(q^{\prime}+q\right) / 2\right)$. Then an epimorphism $\theta: \Lambda \rightarrow G$ defined by the assignment: $\theta\left(e_{i}\right)=1$ for $i=2, \ldots, p-1$, $\theta\left(e_{1}\right)=\theta\left(e_{p}\right)=a b, \theta\left(e_{p+1}\right)=(a b)^{r}$ and

$$
\theta\left(c_{i 0}\right)= \begin{cases}a & \text { for } i=1, \ldots,(p-1) / 2+\left(q^{\prime}-q\right) / 4, \text { and } i=p, \\ b & \text { for } i=(p-1) / 2+\left(q^{\prime}-q\right) / 4+1, \ldots, p-1,\end{cases}
$$

gives rise to a configuration of $(M-q)$ - and $\left(M-q^{\prime}\right)$-symmetries whose product is a $p$-hyperelliptic involution. Both symmetries are non-separating by Lemma 3.2 , since $\theta\left(e_{1}\right)=a b$.

If $q=q^{\prime}$ and $p$ is odd consider an NEC-group $\Lambda$ with the signature

$$
\left(0 ;+;[2, . r ., 2] ;\left\{(-)^{p-1},(2,2),(2, . s ., 2)\right\}\right)
$$

where $r=q-(p+1), s=2(g+1-p-q)$. Then an epimorphism $\theta: \Lambda \rightarrow G$ defined by the assignment: $\theta\left(e_{i}\right)=1$ for $i=1, \ldots, p, \theta\left(e_{p+1}\right)=(a b)^{r}$ and

$$
\theta\left(c_{i 0}\right)= \begin{cases}a & \text { for } i=1, \ldots,(p-1) / 2, \\ b & \text { for } i=(p-1) / 2+1, \ldots, p-1\end{cases}
$$

gives rise to a configuration of two $(M-q)$-symmetries whose product is a $p$-hyperelliptic involution. If $q$ is odd, both symmetries are nonseparating, since in this case $r>0$ as $p \leq q-2<q-1$. Also if $q$ is even 
and $p<q-1$ we have $r>0$. Otherwise if $q=2$ (the case of even $q \geq 4$ was considered in the previous part of the proof) and $p=q-1$ consider

$$
\left(0 ;+;[-] ;\left\{(2,2),\left(2,{ }^{2 g-4} \cdot 2\right)\right\}\right) .
$$

Then an epimorphism $\theta: \Lambda \rightarrow G$ defined by the assignment: $\theta\left(e_{i}\right)=a b$ for $i=1,2$ gives rise to a configuration of two $(M-2)$-symmetries whose product is a 1-hyperelliptic involution. Both symmetries are nonseparating by Lemma 3.2, since $\theta\left(e_{1}\right)=a b$.

Let now $\left(q^{\prime}-q\right) / 2$ and $p$ be odd. Consider an NEC-group $\Lambda$ with signature

$$
\left(0 ;+;[2, . \stackrel{r}{.}, 2] ;\left\{(-)^{p},(2, . s ., 2)\right\}\right)
$$

where $r=\left(q^{\prime}+q\right) / 2-p, s=2\left(g+1-p-\left(q^{\prime}+q\right) / 2\right)$. Then an epimorphism $\theta: \Lambda \rightarrow G$ defined by the assignment: $\theta\left(e_{i}\right)=1$ for $i=$ $1, \ldots, p, \theta\left(e_{p+1}\right)=(a b)^{r}$ and

$$
\theta\left(c_{i 0}\right)= \begin{cases}a & \text { for } i=1, \ldots,(p-1) / 2+\left(q^{\prime}-q-2\right) / 4, \text { and } i=p, \\ b & \text { for } i=(p-1) / 2+\left(q^{\prime}-q-2\right) / 4+1, \ldots, p-1\end{cases}
$$

gives rise to a configuration of $(M-q)$ - and $\left(M-q^{\prime}\right)$-symmetries whose product is a $p$-hyperelliptic involution. Both symmetries are non-separating by Lemma 3.2 , since $r>0$.

Now if $\left(q^{\prime}-q\right) / 2$ is odd and $p$ is even, consider an NEC-group with signature

$$
\left(0 ;+;[2, . r ., 2] ;\left\{(-)^{p},(2, . \stackrel{s}{.}, 2)\right\}\right)
$$

where $r=\left(q^{\prime}+q-2\right) / 2-p, s=2\left(g+2-p-\left(q^{\prime}+q\right) / 2\right)$. Then an epimorphism $\theta: \Lambda \rightarrow G$ defined by the assignment: $\theta\left(e_{i}\right)=1$ for $i=$ $3, \ldots, p, \theta\left(e_{1}\right)=\theta\left(e_{2}\right)=a b, \theta\left(e_{p+1}\right)=(a b)^{r}$ and

$$
\theta\left(c_{i 0}\right)= \begin{cases}a & \text { for } i=1, \ldots, p / 2+\left(q^{\prime}-q-2\right) / 4+1 \\ b & \text { for } i=p / 2+\left(q^{\prime}-q-2\right) / 4+2, \ldots, p\end{cases}
$$

gives rise to a configuration of $(M-q)$ - and $\left(M-q^{\prime}\right)$-symmetries whose product is a $p$-hyperelliptic involution. Both symmetries are non-separating since $\theta\left(e_{2}\right)=a b$.

Let finally $q^{\prime}-q$ be odd. Assume fist that $q^{\prime}-q=4 \alpha+1$ for some integer $\alpha$. Let first $p$ be odd. Consider an NEC-group $\Lambda$ with signature

$$
\left(0 ;+;[2, . \stackrel{r}{.}, 2] ;\left\{(-)^{p},(2, . \stackrel{s}{.}, 2)\right\}\right)
$$

where $r=\left(q^{\prime}+q-1\right) / 2-p, s=2\left(g+1-p-\left(q^{\prime}+q-1\right) / 2\right)$. Then an epimorphism $\theta: \Lambda \rightarrow G$ defined by the assignment: $\theta\left(e_{i}\right)=1$ for 
$i=2, \ldots, p, \theta\left(e_{1}\right)=a b, \theta\left(e_{p+1}\right)=(a b)^{r}$ and

$$
\theta\left(c_{i 0}\right)= \begin{cases}a & \text { for } i=1, \ldots,(p-1) / 2+\left(q^{\prime}-q-1\right) / 4+1, \\ b & \text { for } i=(p-1) / 2+\left(q^{\prime}-q-1\right) / 4+2, \ldots, p,\end{cases}
$$

gives rise to a configuration of $(M-q)$ - and $\left(M-q^{\prime}\right)$-symmetries whose product is a $p$-hyperelliptic involution. Both symmetries are non-separating as above by Lemma 3.2, since $\theta\left(e_{1}\right)=a b$.

If $p$ is even, then with the same group $\Lambda$ as before, consider an epimorphism $\theta: \Lambda \rightarrow G$ defined by the assignment: $\theta\left(e_{i}\right)=1$ for $i=2, \ldots, p$, $\theta\left(e_{1}\right)=a b, \theta\left(e_{p+1}\right)=(a b)^{r}$ and

$$
\theta\left(c_{i 0}\right)= \begin{cases}a & \text { for } i=1, \ldots, p / 2-\left(q^{\prime}-q-1\right) / 4, \\ b & \text { for } i=p / 2-\left(q^{\prime}-q-1\right) / 4+1, \ldots, p .\end{cases}
$$

It is easy to see that this epimorphism gives rise to a configuration of $(M-q)$ - and $\left(M-q^{\prime}\right)$-symmetries whose product is a $p$-hyperelliptic involution. Both symmetries are non-separating since $\theta\left(e_{1}\right)=a b$.

Assume now that $q^{\prime}-q=4 \alpha+3$ for some integer $\alpha$ and let $p$ be even. Consider an NEC-group with signature

$$
\left(0 ;+;[2, . \stackrel{r}{.}, 2] ;\left\{(-)^{p},(2, . \stackrel{s}{.}, 2)\right\}\right)
$$

where $r=\left(q^{\prime}+q-1\right) / 2-p, s=2\left(g+1-p-\left(q^{\prime}+q-1\right) / 2\right)$. Then an epimorphism $\theta: \Lambda \rightarrow G$ defined by the assignment: $\theta\left(e_{i}\right)=1$ for $i=2, \ldots, p, \theta\left(e_{1}\right)=a b, \theta\left(e_{p+1}\right)=(a b)^{r}$ and

$$
\theta\left(c_{i 0}\right)= \begin{cases}a & \text { for } i=1, \ldots,(p-2) / 2+\left(q^{\prime}-q-3\right) / 4+2, \\ b & \text { for } i=(p-2) / 2+\left(q^{\prime}-q-3\right) / 4+3, \ldots, p\end{cases}
$$

gives rise to a configuration of $(M-q)$ - and $\left(M-q^{\prime}\right)$-symmetries whose product is a $p$-hyperelliptic involution. Both symmetries are non-separating by Lemma 3.2 , as $\theta\left(e_{1}\right)=a b$.

Finally assume now that $p$ is odd. Let $\Lambda$ be an NEC-group with signature

$$
\left(0 ;+;[2, . r ., 2] ;\left\{(-)^{p},(2, . s ., 2)\right\}\right)
$$

where $r=\left(q^{\prime}+q-1\right) / 2-p, s=2\left(g+1-p-\left(q^{\prime}+q-1\right) / 2\right)$. Then an epimorphism $\theta: \Lambda \rightarrow G$ defined by the assignment: $\theta\left(e_{i}\right)=1$ for $i=1, \ldots, p-1, \theta\left(e_{p}\right)=a b, \theta\left(e_{p+1}\right)=(a b)^{r}$ and

$$
\theta\left(c_{i 0}\right)= \begin{cases}a & \text { for } i=1, \ldots,(p-3) / 2+\left(q^{\prime}-q-3\right) / 4+2, \\ b & \text { for } i=(p-3) / 2+\left(q^{\prime}-q-3\right) / 4+3, \ldots, p,\end{cases}
$$


gives rise to a configuration of $(M-q)$ - and $\left(M-q^{\prime}\right)$-symmetries whose product is a $p$-hyperelliptic involution. As above both symmetries are non-separating by Lemma 3.2 , since $\theta\left(e_{p}\right)=a b$.

\section{Some consequences and corollaries}

Theorem 4.1. A Riemann surface $X$ of genus $g>4 q+1$ has at most two $(M-q)$-symmetries and their product is the p-hyperelliptic involution for some $p$ with $0 \leq p \leq 2[q / 2]$.

Proof: Assume that $a_{1}, a_{2}, a_{3}$ are $(M-q)$-symmetries of $X$. Then by Propositions 3.4-3.6, $a_{1} a_{2}$ is a $p$-hyperelliptic involution and $a_{1} a_{3}$ is a $p^{\prime}$-hyperelliptic involution for some $p$ and $p^{\prime}$ in range $0 \leq p, p^{\prime} \leq 2[q / 2]$. Now Tyszkowska [8] has shown that if $a_{1} a_{2} \neq a_{1} a_{3}$ then $g \leq 2 p+2 p^{\prime}+1$. However in our case $g>4 q+1 \geq 2 q+2 q+1 \geq 2 p+2 p^{\prime}+1$. So $a_{1} a_{2}=a_{1} a_{3}$ which gives $a_{2}=a_{3}$.

Recall that the hyperelliptic involution is the $p$-hyperelliptic involution with $p=0$.

Corollary 4.2 (Natanzon [6]). A Riemann surface $X$ of genus $g \geq 2$ has at most two $M$-symmetries and their product is the hyperelliptic involution.

Corollary 4.3 (Natanzon [6]). A Riemann surface $X$ of genus $g, g>5$ has at most two $(M-1)$-symmetries and their product is the hyperelliptic involution.

Theorem 4.4. Let $X$ be a p-hyperelliptic Riemann surface of genus $g$, $g>4 p+1$ admitting an $(M-q)$-symmetry for $g \geq 2 q+1$. Then $X$ admits another $\left(M-q^{\prime}\right)$-symmetry for some $q^{\prime}$ in range $q-2 p \leq q^{\prime} \leq q+2 p$.

Proof: Let $a$ be an $(M-q)$-symmetry and let $\rho$ be a $p$-hyperelliptic involution. For $g>4 p+1, \rho$ is unique by [5] and so the group $G$ generated by $\rho$ and $a$ is isomorphic to $\mathrm{Z}_{2} \oplus \mathrm{Z}_{2}$. Then $G=\Lambda / \Gamma$ for some NEC-group with signature (3).

Since $p=\varepsilon h+k+l-1$ we have $p \geq l-1$. But for $p=l-1, k=0$ and $g>4 p+1=4 l-3$ which in turn gives $l<(g+3) / 4$. So $a$ has less than $(g+3) / 2$ ovals and therefore $g+1-q<(g+3) / 2$. This gives $g<2 q+1$ which is not our case.

So $p \geq l$ and thus since an empty period cycle contributes to $a$ or $a \rho$ with at most 2 ovals, we have, by Lemma 3.1, $\|\mid a\|-\|a \rho\| \| \leq 2 l \leq 2 p$ (where $\|\cdot\|$ denotes the number of ovals of a symmetry) and therefore $a \rho$ is an $\left(M-q^{\prime}\right)$-symmetry for $q^{\prime}$ in range $q-2 p \leq q^{\prime} \leq q+2 p$. 
Corollary 4.5 (Natanzon [6]). Let $X$ be a hyperelliptic Riemann surface of genus $g$, admitting an $M$-symmetry. Then $X$ admits another $M$-symmetry.

Corollary 4.6 (Natanzon $[6])$. Let $X$ be a hyperelliptic Riemann surface of genus $g, g \geq 3$, admitting an $(M-1)$-symmetry. Then $X$ admits another $(M-1)$-symmetry.

Theorem 4.7. Let $X$ be a Riemann surface of genus $g$ which has exactly one $(M-q)$-symmetry for $g>2 q+1$ and is not p-hyperelliptic for $g<4 p+1$. Then $X$ does not have fixed point free symmetries.

Proof: Let $a$ be an $(M-q)$-symmetry and let $b$ be arbitrary symmetry of $X$. Clearly $b a b$ is an $(M-q)$-symmetry and so $b a b=a$. Then $G=\langle a, b\rangle=\mathrm{Z}_{2} \oplus \mathrm{Z}_{2}=\Lambda / \Gamma$ for some NEC-group $\Lambda$, say with signature (3). Now the epimorphism $\theta: \Lambda \rightarrow G$ sends the canonical reflections corresponding to nonempty period cycle alternatively to $a$ and $b$. So, by Lemma 2.1, the proof will be finished if we show that $k>0$. Let us observe first that $X$ is $p$-hyperelliptic for $p=\varepsilon h+l+k-1$. So $(g-1) / 4 \geq p \geq l-1$ which gives $l \leq(g+3) / 4$. Therefore, by Lemma 2.1, for $k=0$, we have $g+1-q \leq(g+3) / 2$. This gives $g \leq 2 q+1$ which is not our case.

\section{References}

[1] E. Bujalance, A. F. Costa and D. Singerman, Application of Hoare's theorem to symmetries of Riemann surfaces, Ann. Acad. Sci. Fenn. Ser. A I Math. 18(2) (1993), 307-322.

[2] E. Bujalance And A. F. Costa, A combinatorial approach to the symmetries of $M$ and $M-1$ Riemann surfaces, in: "Discrete groups and geometry" (Birmingham, 1991), London Math. Soc. Lecture Note Ser. 173, Cambridge Univ. Press, Cambridge, 1992, pp. 16-25.

[3] E. Bujalance and A. F. Costa, On symmetries of $p$-hyperelliptic Riemann surfaces, Math. Ann. 308(1) (1997), 31-45.

[4] E. Bujalance, J. J. Etayo, J. M. Gamboa and G. Gromadzki, "Automorphism groups of compact bordered Klein surfaces. A combinatorial approach", Lecture Notes in Mathematics 1439, SpringerVerlag, Berlin, 1990.

[5] H. M. Farkas and I. Kra, "Riemann surfaces", Graduate Texts in Mathematics 71, Springer-Verlag, New York-Berlin, 1980.

[6] S. M. Natanzon, Finite groups of homeomorphisms of surfaces, and real forms of complex algebraic curves, (Russian), Trudy Moskov. 
Mat. Obshch. 51 (1988), 3-53, 258; translation in: Trans. Moscow Math. Soc. (1989), 1-51.

[7] D. Singerman, On the structure of non-Euclidean crystallographic groups, Proc. Cambridge Philos. Soc. 76 (1974), 233-240.

[8] E. Tyszkowska, On $p q$-hyperelliptic Riemann surfaces, Colloq. Math. 103(1) (2005), 115-120.

Institute of Mathematics

Gdańsk University

Wita Stwosza 57

80-952 Gdańsk

Poland

E-mail address: retrakt@math.univ.gda.pl

Primera versió rebuda el 31 de març de 2006, darrera versió rebuda el 15 de març de 2007. 